\title{
4-Point Minimal Pick \& Place Trajectory Design in Robotics
}

\author{
Satvik M. Kusagur, Arun Kumar G., Spoorthi Jainar, T.C.Manjunath, Pavithra G.
}

\begin{abstract}
Motion planning in robotics plays a very important role in the movement of objects from the source to the destination. Robots are classified according to motion control as PNP, PTP and $C P$ robots. Hence, there are 3 basic types of trajectory motions +1 trajectory which is the shortest path between two points in the $3 D$ space. This type of motion or trajectory is exhibited by PNP robots. In this paper, we develop the theoretical background along with the mathematical formulation relating to the design \& development of a 4-point minimal pick \& place trajectory from the source to the destination during the transportation of an object in the 3 dimensional Euclidean space R3.
\end{abstract}

Index Terms - Robot, Motion planning, Trajectory, Source, Destination, 4-Point, Pick, Lift-off, Set-down, Place, Obstacle.

\section{ORganization OF THE RESEARCH ARTICLE}

Any typical robotic operation begins with the robot picking up the object. The main function of any robotic manipulator is to pick up an object from one particular place which is in one particular position and orientation and keep it at another particular place in another position and orientation. Robot picks up an object from one place, moves along a specified path and keeps it at another place in a specified time, thus giving rise to a trajectory. The trajectory obtained so is called PNP trajectory. The pick and place points has to be explicitly specified by the user, whereas the path can be specified by the user or the robot can judge its own path using computer vision. The path that is taken by the robot to reach the destination may be the shortest one or longest one irrespective of overcoming the obstacles in its path of motion and preventing collisions with them. This operation is called as PNPO ( Pick aNd Place Operation) [2] [8].

The path or route that is taken by the robot from the pick point to the place point with the time information is called as the four point minimal pick and place trajectory. Any PNP trajectory will have four points passing through it which are discussed in greater detail one below the other. They are [1]

(1) Pick point.

(2) Lift-off point.

(3) Via points or intermediate points

(4) Set-down point.

(5) Place point.
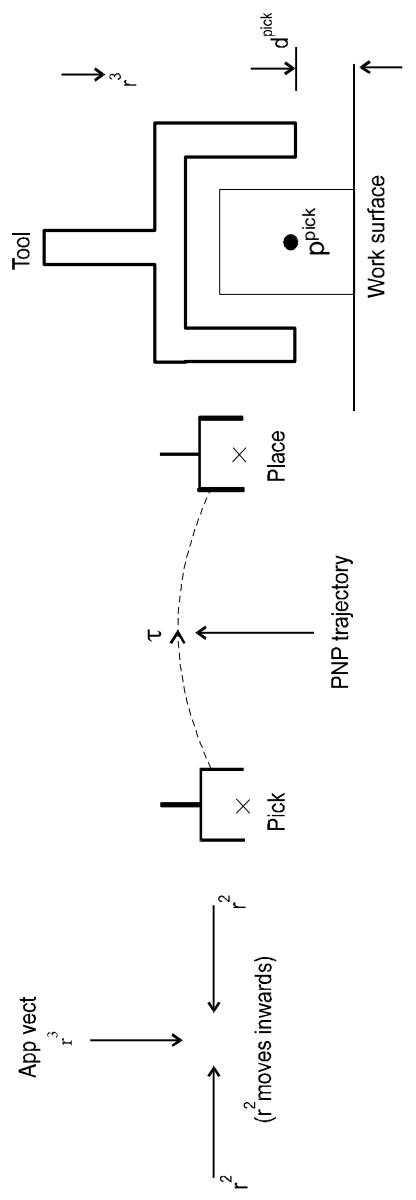

Fig. 1 : Pick point

\section{Generation Of The Pick Point $P^{\text {PICK }}$}

It is the first point in the PNP trajectory. It is the point from where the object or the part has to be picked up and represents the initial position of the part and is given by $p^{\text {pick }}$. The object has to be picked up about its centroid $\mathrm{G}$ using fine motion, i.e., tool-tip p of the gripper and centroid of the object $\mathrm{G}$ of the object should be the same. The object has to be picked up about its centroid for efficient grasping. The gripping force $f_{g}$ should be such that $f_{g}>$ (weight of the object $\mathrm{w}+$ the gravitational pull $\mathrm{g}$ ), otherwise the object falls down from within the fingers [3] [4]. 


\section{4-point minimal pick \& place trajectory design in robotics}

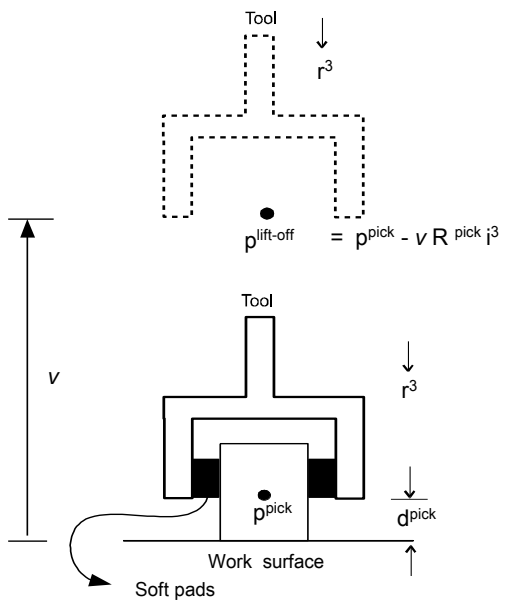

Fig. 2 : Lift-off operation

The object is picked up using the approach vector $r^{3} \perp^{r}$ work surface $\mathrm{x}^{0} \mathrm{y}^{0}$ on which the part is resting and the sliding vector $r^{2}$ moving inwards along the closing axis of gripper or tool such that $r^{2}=-i^{2}$ as shown in Fig. 2, where $i$ is a unit vector. $d{ }^{\text {pick }}$ represents the vertical distance from the horizontal work surface to the pick point or center of the object [2] [8]. Remember, if a part has to be picked up ; then, the position and orientation of the part at the pick event has to be specified in the form of a matrix. The object is picked up using the fine motion [5][6].

The position and orientation of the pick operation is given by a HCTM $\mathrm{T}^{\text {Pick }}$ which is given by

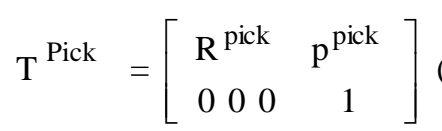

where $\mathrm{R}^{\text {pick }}$ is the orientation of the tool at the pick point and $\mathrm{p}^{\text {pick }}$ is the position of the pick point. To this pick point, one RHOCF is attached. $\mathrm{p}^{\text {pick }}$ and $\mathrm{R}^{\text {pick }}$ are to be specified by the user or can be obtained automatically using robot vision. When this matrix is inputted to the IKP, sets of joint variables will the calculated and the tool will be properly configured so as to come and pick up the object [7] [8] [9].

\section{GENERATION OF THE LIFT-OFF POINT $P^{\text {LIFT-OFF }}$}

It is the second point in the PNP trajectory. It is a point very near to pick point and is situated directly above the pick point by a small amount of distance of $v$ units and is given by $\mathrm{p}^{\text {lift-off. }}$. It is the point where the object is lifted up from the pick point using the fine motion and is shown in Figs. $1 \& 2$. Remember, if a part has to be lifted up ; then, the position and orientation of the part at the lift-off point has to be specified in the form of a matrix. The position and orientation of the lift-off operation is given by a HCTM,

$$
\mathrm{T}^{\text {Lift-off }}=\left[\begin{array}{ccc}
\mathrm{R}^{\text {lift-off }} & \mathrm{p}^{\text {lift-off }} \\
0 & 0 & 0
\end{array}\right]
$$

where $\mathrm{R}^{\mathrm{Lift}-\text { off }}$ is the orientation of the tool at the lift-off point and $\mathrm{p}^{\text {lift-off }}$ is the position of the lift-off point. To this lift-off point, one RHOCF is attached. $\mathrm{p}^{\text {lift-off }}$ and $\mathrm{R}^{\text {lift-off }}$ are to be specified by the user or can be obtained automatically using robot vision. The orientation of the tool at lift-off point is the same as that of the pick point, i.e., $\mathrm{R}^{\text {lift-off }}=\mathrm{R}^{\text {pick }}$. But, the position of the lift-off point is obtained by moving backwards along the approach vector $\mathrm{r}^{3}$ from $\mathrm{p}^{\text {pick }}$ by a small amount of distance $v$ units. The position of the lift-off point is given by $\mathrm{p}^{\text {lift-offf }}=\mathrm{p}^{\text {pick }}-v \mathrm{R}^{\text {pick }} \mathrm{i}^{3}$. The $\mathrm{p}$ and $\mathrm{R}$ at lift-off is given by [10]-[12]

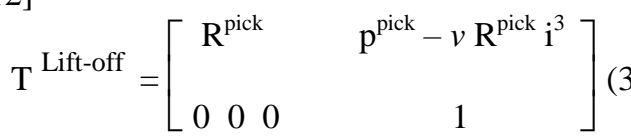

When this matrix is inputted to the IKP, set of joint variables will the calculated, the robot comes from the home position, picks up the object using fine motion and exactly lifts it up using the fine motion and stops at the lift-off point. The position of the lift-off coordinate frame is determined by using the equation $\mathrm{p}^{\text {wrist }}=\mathrm{p}-\mathrm{d}_{\mathrm{n}} \mathrm{r}^{3} \cdot \mathrm{p}^{\text {lift-offf }}=\mathrm{p}^{\text {pick }}-v \mathrm{R}^{\text {pick }} \mathrm{i}^{3}$; In this equation, $\mathrm{p}^{\text {pick }}$ is a $(3 \times 1)$ vector, $v$ is a scalar, $\mathrm{R}^{\text {pick }}$ is a $(3 \times 3)$ matrix, $i^{3}$ is a unit vector along the $\mathrm{z}$-axis given by [ $\left.\begin{array}{lll}0 & 0 & 1\end{array}\right]^{\mathrm{T}}$. It is like subtracting a $(3 \times 1)$ vector from another ( $3 \times 1$ ) vector multiplied by a scalar $v[13]-[15]$.

\section{Generation OF THE SET-Down PoInT $\mathrm{P}^{\text {SET-Down }}$}

It is the $3^{\text {rd }}$ point in the PNP trajectory and is analogous to the lift-off point. It is a point very near to the place point and is situated directly above the place point by a small amount of distance of $v$ units and is given by $\mathrm{p}^{\text {set-down }}$. Set-down point is defined as the point where the initiation of the object placement just begins [16] - [17].

The tool reaches the set-down point from the lift-off point using the gross motion and is about to place the object at the place position using fine motion. Remember, if a part has to reach the set-down point ; then, the position and orientation of the part at the set-down has to be specified in the form of a matrix. The position and orientation of the set-down operation is given by a HCTM [18] - [20] [2] [8]

$$
\mathrm{T}^{\text {set-down }}=\left[\begin{array}{cccc}
\mathrm{R}^{\text {Set-down }} & \mathrm{p}^{\text {Set-down }} \\
0 & 0 & 0 & 1
\end{array}\right] \text { (4) }
$$

where $\mathrm{R}^{\text {set-down }}$ is the orientation of the tool at the set down point and $\mathrm{p}^{\text {set-down }}$ is the position of the tool at the set-down point [21] - [23] [2] [8].

To this set-down point, one RHOCF is attached. $\mathrm{p}^{\text {Set-down }}$ and $\mathrm{R}^{\text {Set-down }}$ are to be specified by the user or can be obtained automatically using robot vision [24] - [26].

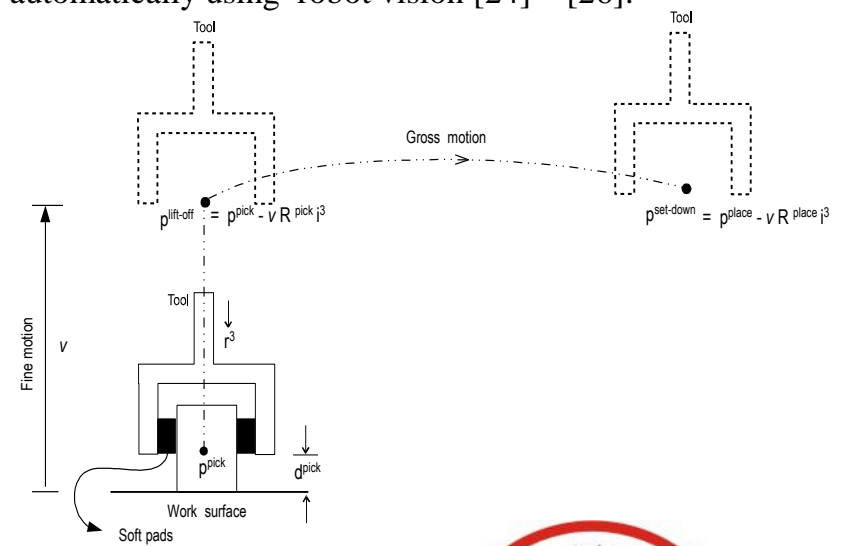

Fig. 3 : Object being lifted from pick to set-down point 
The orientation of the tool at the set-down point is the same as that of the place point, i.e., $\mathrm{R}^{\text {Set-down }}=\mathrm{R}^{\text {Place }}$. But, the position of the set-down point is obtained by moving backwards along the approach vector $\mathrm{r}^{3}$ from $\mathrm{p}^{\text {place }}$ by a small amount of distance $v$. The position of the set-down point is given by $\mathrm{p}^{\text {Set-down }}=\mathrm{p}^{\text {Place }}-v \mathrm{R}^{\text {Place }} \mathrm{i}^{3}$. The $\mathrm{p}$ and $\mathrm{R}$ at set-down point is given by [27] - [30] [2] [8]

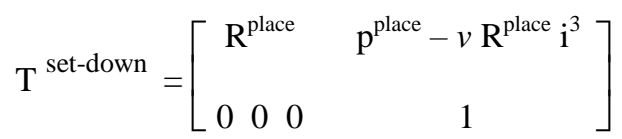

When this matrix is inputted to the IKP, sets of joint variables will the calculated, the robot comes from the home position, picks up the object using fine motion and exactly lifts it up using the fine motion and reaches the set-down point using the gross motion. The position of the set-down coordinate frame is determined by using the equation [31]

$$
\mathrm{p}^{\text {wrist }}=\mathrm{p}-\mathrm{d}_{\mathrm{n}} \mathrm{r}^{3}
$$

\section{Generation of the Place Point P $P^{\text {Place }}$}

It is the $4^{\text {th }}$ and last point or the final point in the PNP trajectory and is given by $\mathrm{p}^{\text {place }}$. The place point is defined as the point where the object has to be placed in the desired position and orientation using the fine motion. The object is placed with the approach vector $\mathrm{r}^{3} \perp^{\mathrm{r}}$ work surface $\mathrm{x}^{0} \mathrm{y}^{0}$ on which the part is about to be placed and with the sliding vector $r^{2}$ moving outwards (i.e., along the opening axis of the gripper or tool such that $r^{2}=+i^{2}$ as shown in Fig. 4, where $i$ is a unit vector) [32] $-[33]$.

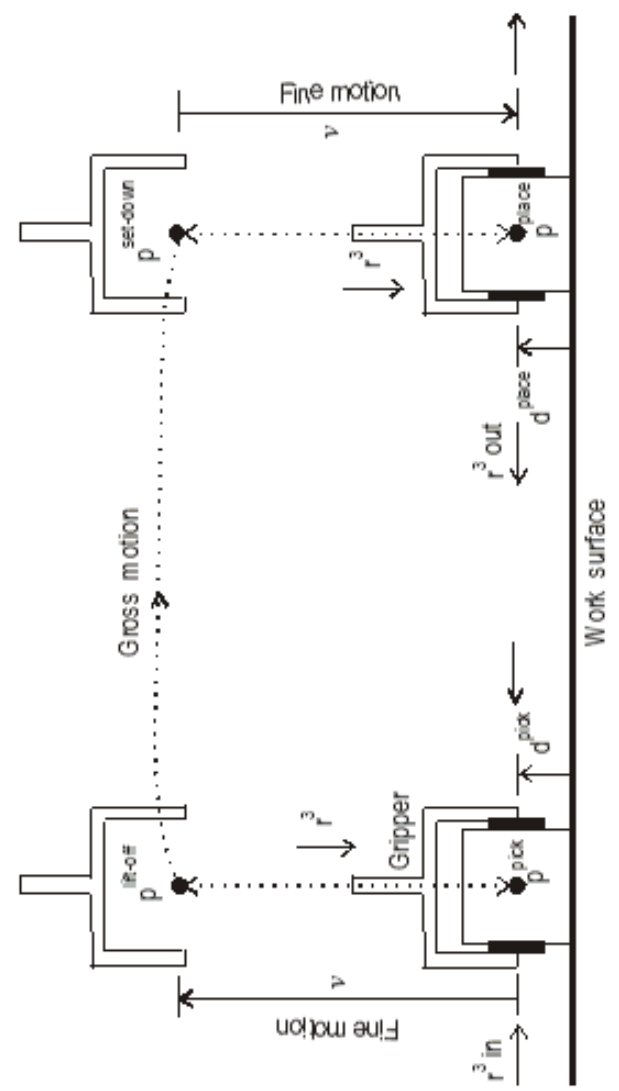

Fig. 4 : Object being placed at the placed point from the set-down point

$\mathrm{d}^{\text {Place }}$ represents the vertical distance from the horizontal work surface to the centroid of the gripper or the centre of the object at the place point as shown in the Fig. 4 [2] [8]. Remember, if a part has to be placed on the work surface ; then, the position and orientation of the part at the place point has to be specified in the form of a matrix. The position and orientation of the place operation is given by a HCTM [34] [2] [8]

$$
\mathrm{T}^{\text {place }}=\left[\begin{array}{cccc}
\mathrm{R}^{\text {place }} & \mathrm{p}^{\text {place }} \\
0 & 0 & 0 & 1
\end{array}\right] \text { (7) }
$$

where $\mathrm{R}^{\text {place }}$ is the orientation of the tool at the place point and $\mathrm{p}^{\text {Place }}$ is the position of the tool at the place point. To this place point, one $\mathrm{RHOCF}$ is attached. $\mathrm{p}^{\text {Place }}$ and $\mathrm{R}^{\text {Place }}$ are to be specified by the user or can be obtained automatically using robot vision [2] [8].

When this matrix is inputted to the IKP, set of joint variables will the calculated, the robot comes from the home position, picks up the object using fine motion and exactly lifts it up using the fine motion, reaches the set-down point using the gross motion and places the object at the place position in the desired position and orientation using the fine motion [2] [8].

$\mathrm{d}^{\text {Place }}$ represents the vertical distance from the horizontal work surface to the centroid of the gripper or the centre of the object at the place point as shown in the Fig. 4 [2] [8]. Remember, if a part has to be placed on the work surface ; then, the position and orientation of the part at the place point has to be specified in the form of a matrix. The position and orientation of the place operation is given by a HCTM [34] [2] [8]

\section{Effective Pick \& Place Constraints for doIng AN PNPO OPERATION (PICK \& PlaCE OPERATION)}

If all the 4 matrices, viz., $\mathrm{T}^{\text {Pick }}, \mathrm{T}^{\text {Lift-off }}, \mathrm{T}^{\text {set-down }}$ and $\mathrm{T}^{\text {place }}$ are given in succession one after another to the robot then, the robot moves from the home position using the fine motion and gross motion, reaches the lift-off point using the gross motion, moves upto the pick point using the fine motion, picks up the object using fine motion, lifts it up using the fine motion, transports the part from the lift-off point to the set-down point using gross motion, gets ready to place the object and places the object in the desired position and orientation using fine motion [2] [8].

Then, opens its fingers, moves backwards upto the set-down point using fine motion and then comes back to the home position using gross motion and fine motion. These events of operations is shown in Fig. 7. If any obstacles are present in the work space, a number of via points (intermediate points) are used to move round the obstacles in order to avoid collision by sensing them and moving to the destination as shown in Fig. 4. During a PNP trajectory, lift-off point and set-down point is visited twice whereas other points are visited only once. Let [2] [8]

$\mathrm{d}^{\text {place }}=$ Distance of the place point $\mathrm{p}$ from the work surface along the approach vector $r^{3}$. 
$\mathrm{d}^{\text {pick }}=$ Distance of the pick point $\mathrm{p}$ from the work surface along the approach vector $\mathrm{r}^{3}$.

3 important PNP constraints for doing effective PNP operation are shown in the Fig. 5 \& depicted in meaning as above [2] [8].

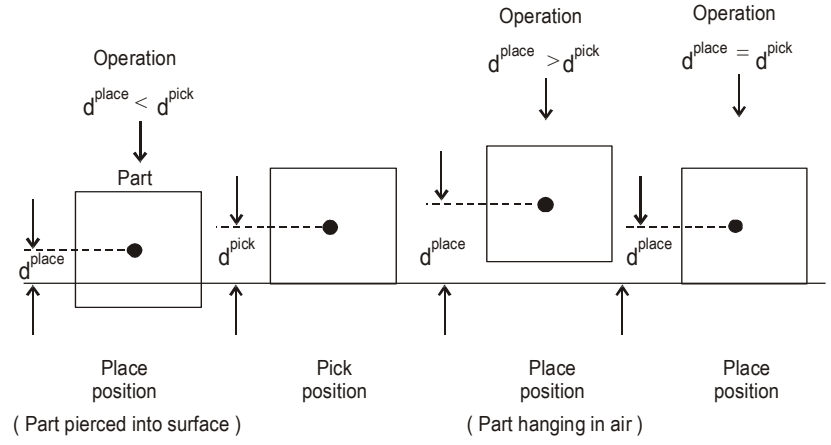

Fig. 5 : Constraints on pick and place positions

\section{CONSTRAINTS ON PICK AND PLACE POSITIONS}

It has to be noted that there are 3 constraints on the pick $\&$ place positions during the transportation of an object from the pick point to the place point, they are [2] [8]

- If $\mathrm{d}^{\text {place }}=\mathrm{d}^{\text {pick }}$; Object is picked up from the work surface and placed exactly touching the work surface.

- If $\mathrm{d}^{\text {place }}<\mathrm{d}^{\text {pick }}$; An attempt is made by the robot to penetrate the object into the work surface when part is placed, as a result of which the part slides in between the fingers of the gripper if the work surface is hard OR the part moves into the work surface if the work surface is soft.

- If $\mathrm{d}^{\text {place }}>\mathrm{d}^{\text {pick }}$; The placed part or object is unsupported (hanging in air) when it reaches the destination and the robot opens its fingers and the object or the part falls down because of gravity (some inaccuracy has been resulted) [2] [8]. the effective PNP constraint is

$\mathrm{d}^{\text {place }}=\mathrm{d}^{\text {pick }}$

$\mathrm{p}^{\text {place }}=\mathrm{p}^{\text {pick }}(9)$

and is shown in Fig. 5

\section{Four POINT MINIMAL PNP TRAJECTORY}

The sequence of PNP motions shown in Fig. 7 is called as the minimal four point PNP trajectory. Any PNP trajectory has got 6 knot points ( pick, lift-off, via 1, via 2, set-down, place ) and 5 individual sub-trajectories ( pick-lift off; lift off-via 1 ; via 1 -via 2 ; via 2 -set down; set down-place). A operation. If part feeders etc., are used, then use different PNP surfaces or levels and the height of the stack comes into picture [2] [8].

- From the PNP trajectory, we see that robot reaches or approaches the object using fine motion and picks it up. operation given by $\mathrm{T}^{\text {Pick }}$.
Therefore, in order to do a successful PNPO by the robot, horizontal work surface is assumed for doing the PNP

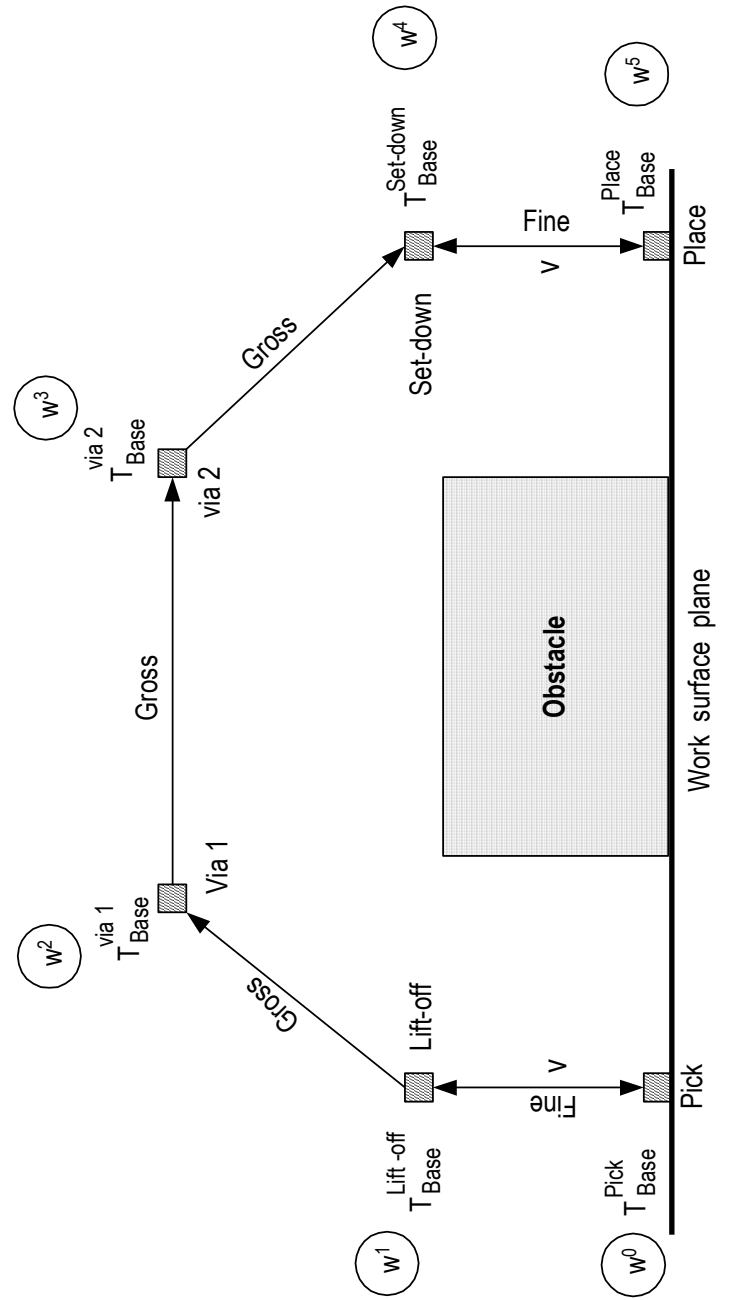

Fig. 7 : A four point minimal PNP trajectory consisting of 6 knot points and 5 individual sub-trajectories

Table 1 : Motion type for a PNP operation

\begin{tabular}{|l|l|l|}
\hline Destination & $\begin{array}{l}\text { Type of } \\
\text { Motion }\end{array}$ & Speed \\
\hline Lift-off & Gross & Fast \\
Pause & Fine & Zero \\
Pick & Fine & Very slow \\
Grasp & Fine & Slow \\
Lift-off & Fine & Slow \\
Via & Gross & Fast \\
Set-down & Gross & Fast \\
Pause & Fine & Zero \\
Place & Fine & Very slow \\
Release & Fine & Slow \\
Set-down & Fine & Slow \\
\hline
\end{tabular}

- Using fine motion technique, object or part is lifted from the pick up point to the lift-off point.

..... operation given by the matrix $\mathrm{T}^{\text {Lift-off }}$.

- Using gross motion, object or part is transported to the set-down point.

operation given by $\mathrm{T}^{\text {Set-down }}$.

- During this transportation, if any obstacles occur or come in the way of robot motion, a number of via points or intermediate points has to be visited in order to over come the obstacle (move round the 
obstacle, circumvent the obstacle). given by $\mathrm{T}^{\text {via } 1}$ and $\mathrm{T}^{\mathrm{via} 2}$.

- At set-down point, the robot is about to place the object, i.e., the initiation of the object placement just begins.

- The robot places the object at the place point using fine motion techniques in the desired $\mathrm{p}$ and $\mathrm{R}$ and then comes back to its home position.

...... operation represented by $\mathrm{T}^{\text {Place }}$.

The table 1 shown below gives the speed variation for the robot to move from the source to the destination [2] [8].

\section{Conclusions}

The theoretical concepts of a 4-point minimal pick \& place trajectory was developed in this paper along with the mathematical formulations. For the robot to do a particular task, speed is one of the important specification that is required; for example, if the robot is at one particular location and the job / task has to be performed at another location; then, the robot should reach that particular location first. Then, it should make use of the trapezoidal speed profile curve as shown in Fig. 7, i.e., slow at start, accelerate, run at constant speed and decelerate before coming to stop. From home position to the vicinity of the object, gross or coarse motion can be used. As the arm nears the object or part, fine motion can be used. Thus, the robot controller has to be designed in such a way so as to control the speed.

For example; in case of spray painting operations, controlled motions of the tool is very much essential, otherwise the spray painting will be uneven. The speed control can be implemented by software or hardware or both using hardware and software. Gross motion can be carried out at high speed, so that the maximum distance from source to goal is covered and stresses are reduced in the system, since the on time of the actuators is also reduced.

Fine motion can be carried out at reduced speed for accurate positioning control. For example, when robot is approaching the work surface or doing a picking operation or doing a placing operation or a nut mating operation. Sudden increase or decrease in speed can cause overshoot of pick point or place point with the result, object is being penetrated onto the work surface or the fingers getting damaged. Hence, pauses are introduced between points.

Pauses are introduced before fine motion starts as shown in the Table 1. When motion type changes from gross to fine, sudden decrease in speed occurs. Similarly, when motion type changes from fine to gross, sudden increase in speed occurs. A pause (wait state) is introduced in between these two type of motions. This sudden change in speed causes vibrations or oscillations at destination. If pauses are introduced, then these oscillations will die due to zero (this process is called as ringing).

\section{A C K N O W LED G M E N T}

The people responsible for the creation of this paper likes to thank all the officials of JSS Academy of Tech. Edn., Noida, UP \& DSCE in all respects for helping them to publish the paper.

\section{REFERENCES}

[1] Craig J, "Introduction to Robotics : Mechanics", Dynamics \& Control, Addison Wessely, USA, 1986.

[2] Robert J.S., "Fundamentals of Robotics - Analysis and Control", PHI, New Delhi.

[3] Klafter, Thomas and Negin, "Robotic Engineering", PHI, New Delhi.

[4] Fu, Gonzalez and Lee, "Robotics: Control, Sensing, Vision and Intelligence", McGraw Hill, Singapore.

[5] Groover, Weiss, Nagel and Odrey, "Industrial Robotics", McGraw Hill.

[6] Ranky P.G., C.Y. Ho, "Robot Modeling, Control \& Applications", IFS Publishers, Springer, UK.

[7] Crane, Joseph Duffy, "Kinematic Analysis of Robotic Manipulators", Cambridge Press, UK

[8] Manjunath, T.C., "Fundamentals of Robotics", Fifth edn., Nandu Publishers, Mumbai, India, 2007.

[9] Ranky P G and C Y Ho, Robot Modeling, control and applications, IFS publishers, Springer, UK.

[10] Asada, H., and J.E. Slotine, Robot Dynamics \& Control, Wiley, NY.

[11] Phillip Coiffette, Robotics Series (Vol. I to VIII ), Kogan Page, London.

[12] Mohsen Shahinpoor, "Robotic Engineering Text Book", Harper \& Row Publishers.

[13] Janakiraman, "Robotics and Image Processing", Tata McGraw Hill

[14] Richard A Paul, "Robotic Manipulators", MIT press, Cambridge.

[15] Fairhunt, "Computer Vision for Robotic Systems", CFS Pubs., New Delhi.

[16] Yoram Koren, "Robotics for Engineer", McGraw Hill.

[17] Bernard Hodges, "Industrial Robotics", Jaico Publishing House, India.

[18] Tsuneo Yoshikawa, "Foundations of Robotics : Analysis and Control", $P H I$, India.

[19] Dr. Jain and Dr. Aggarwal, "Robotics : Principles \& Practice", Khanna Publishers, Delhi.

[20] Lorenzo and Siciliano, "Modeling and Control of Robotic Manipulators", McGraw Hill.

[21] Dr. Amitabha Bhattacharya, "Mechanotronics of Robotics Systems", Kaizen Publishing, Calculatta, India.

[22] S.R. Deb, "Industrial Robotics", Tata MacGraw Hill..

[23] Edward Kafrissen and Mark Stephans, "Industrial Robots and Robotics", Prentice Hall Inc. , Virginia, USA.

[24] Rex Miller, "Fundamentals of Industrial Robots and Robotics", PWS Kent Pub Co., Boston, USA.

[25] William Burns and Janet Evans, (2000), Practical Robotics - Systems, Interfacing, Applications, Reston Publishing Co.

[26] http://www.wikipedia.org

[27] Michael Dipperstein, Run Length Encoding (RLE) Discussion and Implementation.

[28] Flusser, J.; Suk, T.; Saic, S., Recognition of blurred images by the method of moments, Image Processing, IEEE Transactions.

[29] Bob Bailey, Moments in Image Processing, Nov. 2002.

[30] Phillip Coiffette, (1995), Robotics Series, Volume I to VIII, Kogan Page, London, UK.

[31] Yoshikawa T., (1984), "Analysis and Control of Robot Manipulators with Redundancy", Proc. First Int. Symp. on Robotics Research, Cambridge, MIT Press, pp. 735-748.

[32] Whitney DE., (1972), "The Mathematics of Coordinated Control of Prosthetic Arms and Manipulators", Trans. ASM J. Dynamic Systems, Measurements and Control, Vol. 122, pp. 303-309.

[33] Lovass Nagy V, R.J. Schilling, (1987), "Control of Kinematically Redundant Robots Using \{1\}-inverses", IEEE Trans. Syst. Man, Cybernetics, Vol. SMC-17 (No. 4), pp. 644-649.

[34] Lovass Nagy V., R J Miller and D L Powers, (1978), “An Introduction to the Application of the Simplest Matrix-Generalized Inverse in Systems Science", IEEE Trans. Circuits and Systems, Vol. CAS-25 (No. 9), pp. 776.

[35] Dr. Manjunath, "Modelling \& Control of Smart Structures", Ph.D. Thesis, IIT Bombay, 2007.

[36] Dr. Arunkumar, Ph.D. Thesis, 2016.

\section{AUTHORS PROFILE}

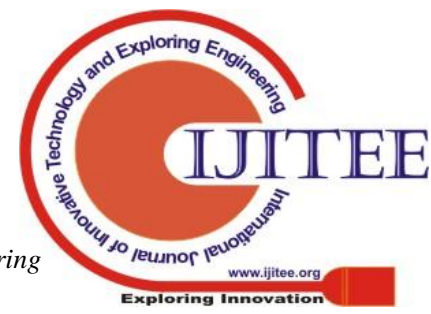


Dr. Arun Kumar G. was born in Davanagere, Karnataka, India on Oct. 15th, 1981 \& received the B.E. Degree (Bachelor of Engg.) from STJ Institute of Technology, Ranebennur in Karnataka in the year 2004, M.Tech. degree in Digital Communication \& Networking from the prestigious UBDT College of Engg., Davanagere in the year 2008 and completed his Ph.D. in Electronics in Visvesvaraya Technological University, Belgaum in the year 2016. He has got a teaching \& administrative experience of more than 12 years in engineering colleges in Karnataka. He has written a number of notes in various subjects as Basic Electronics, AEC, Power Electronics, Communications \& his notes are widely famous all over the country. He has also published 3 text books. Currently, he is working as Associate Professor in the ECE Dept. of JSS Academy of Technical Education @ Noida in Uttar Pradesh, India. He has attended a number of certificate courses, workshops, FDPs, Symposiums, etc. He has published more than 50 papers in various subjects of engineering field. His current areas of interest are control systems, power electronics, basic electronics, micro-controllers, embedded systems, communications etc....

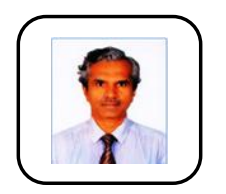

Dr. T.C. Manjunath received the B.E. Degree (Bachelor of Engg. in Electrical Engg.) from R.V. College of Engg. (Bangalore Univ.) in the year 1989, M.E. degree in Automation, Control \& Robotics from the prestigious 100 year old Govt.'s LD College of Engg., (Gujarat Univ., Ahmadabad) in the year 1992 and Ph.D. in Systems \& Control Engineering from the prestigious Indian Institute of Technology Bombay (IIT Bombay) in the year 2007 respectively. He has got a teaching (academic), research \& administrative experience of nearly $32+$ years in various engineering colleges all over the country (Karnataka, Gujarat, Maharashtra). He has published more than $600+$ papers in various National, International Journals and Conferences in India \& abroad and published more than a dozen textbooks. 